\title{
Recent ATC's in the design of Memristors
}

\author{
${ }^{1}$ Medha Haridas, ${ }^{2}$ Shashikanth Patil and ${ }^{3}$ Dr. T.C. Manjunath Ph.D. (IIT Bombay), Member, IEEE \& \\ Fellow IETE, IACST Sr. Member
}

\begin{abstract}
This paper presents a brief review about the recent advanced technology concepts in the field of the 4th element, called as the memristor, which has the properties of $R, L \& C$. Traditionally, there have been three different basic components to circuits, namely capacitors, resistors, and inducers. However, a new basic element to a circuit has been developed. This element is known as the memristor. A memristor is essentially a resistor with memory. The resistance of a memristor is dependent upon the amount of voltage which has been applied to it and the length of time this voltage was applied. The concept of a memristor was first developed in 1971. However, it was not until recently that researchers at HP Labs were able to build the first working one. This is due mostly to the size dependence of the memristor. The changes in resistance of a memristor are not detectable until the memristor is in the nanometer range. When the thickness of the memristor is larger than this, it is indistinguishable from a standard resistor. The main advantage of the memristor in modern computing is that it retains its resistance even when no voltage is applied. This would allow a computer to retain its saved state while off and would require less power to run when it is on. Another advantage to memristors is their analog nature. Whereas the switches in computers have only two states, on and off, the resistance in a memristor does not have a set amount of values it can be. While the memristor can still be used for digital memory storage, there is research currently going on into the development of analog computers using memristors. The readers can use this paper as the ready reckoner for doing their research in the field of memristors, the data being obtained from various sources from the internet $\&$ from the papers of various researchers.
\end{abstract}

Index Terms-Memristor, Resistor, Inductor, SI, Units, Capacitor, Freeze, Memory.

\section{INTRODUCTION}

Memristance is a property of an electronic component. If charge flows in one direction through a circuit, the resistance of that component of the circuit will increase, and if charge flows in the opposite direction in the circuit, the resistance will decrease. If the flow of charge is stopped by turning off the applied voltage, the component will 'remember' the last resistance that it had, and when the flow of charge starts again the resistance of the circuit will be what it was when it was last active. It turns out that memristance is becoming stronger as the feature sizes in circuits are getting smaller. At some point as we scale into the realm of nano-electronics, it

\footnotetext{
${ }^{1}$ Graduate Student, ECE Dept., 8th Semester, New Horizon College of Engg., Bangalore,

${ }^{2}$ Lecturer, ECE Dept., NHCE, B'lore, K'taka, India.

${ }^{3}$ Professor \& Head, Electronics \& Communication Engg. Dept., Rajarajeshwari College of Engg., Bangalore-74, Karnataka, India (emails : tcmanjunath@gmail.com ; tcmanjunath@rediffmail.com).
}

will be necessary to explicitly take account of memristance in our circuit models in order to simulate and design electronic circuits properly [1].

\section{What Is A Memristor ?}

Memristor is a simple word derived from "memory resistor," because that is exactly its function : to remember its history. A memristor is a two-terminal device that is built to express only the properties of memristance. Think of a resistor as a pipe through which water flows. The water is electric charge. The resistor's obstruction of the flow of charge is comparable to the diameter of the pipe: the narrower the pipe, the greater is the resistance. For the history of circuit design, resistors had a fixed pipe diameter. But a memristor is a pipe that changes diameter with the amount and direction of water that flows through it. If water flows through this pipe in one direction, it expands (becoming less resistive). But send the water in the opposite direction and the pipe shrinks (becoming more resistive). Further, the memristor remembers its diameter when water last went through. Turn off the flow and the diameter of the pipe "freezes" until the water is turned back on [2].

\section{IMPORTANCE OF A MEMRISTOR}

It's time to stop shrinking. Moore's Law, the semiconductor industry's obsession with the shrinking of transistors and their commensurate steady doubling on a chip about every two years, has been the source of a 50 year technical and economic revolution. Whether this scaling paradigm lasts for five more years or 15, it will eventually come to an end. The emphasis in electronics design will have to shift to devices that are not just increasingly infinitesimal but increasingly capable. Memristor is the perfect example of such a device [3].

It turns out that memristance is becoming stronger as the feature sizes in circuits are getting smaller. At some point as we scale into the realm of nano-electronics, it will be necessary to explicitly take account of memristance in our circuit models in order to simulate and design electronic circuits properly. Combined with transistors in a hybrid chip, memristors could radically improve the performance of digital circuits without shrinking transistors. Using transistors more efficiently could in turn give us another decade, at least, of Moore's Law performance improvement, without requiring the costly and increasingly difficult doublings of transistor density on chips. In the end, memristors might even become the cornerstone of new analog circuits that compute using architecture much like that of the brain [4]. 


\section{ORIGIN OF MEMRISTOR}

Prof. Leon Chua had just moved to the Electrical Engineering Department of UC Berkeley when he published his seminal paper, "Memristor - The missing circuit element" IEEE Trans. Circuit Theory CT-18, pp. 507-519 (1971). In this paper, Prof. Chua proved a number of theorems to show that there was a 'missing' two-terminal circuit element from the family of "fundamental" passive devices : resistor, capacitor and inductor, for e.g., elements that do not add energy to a circuit [4].

He discovered a missing link in the pair wise mathematical equations that relate the four circuit quantities - charge, current, voltage, and magnetic flux - to one another. These can be related in six ways. Two are connected through the basic physical laws of electricity and magnetism, and three are related by the known circuit elements: resistors connect voltage and current, inductors connect flux and current, and capacitors connect voltage and charge. But one equation is missing from this group : the relationship between charge moving through a circuit and the magnetic flux surrounded by that circuit [5].

Chua demonstrated mathematically that his hypothetical device would provide a relationship between flux and charge similar to what a non-linear resistor provides between voltage and current. In practice, that would mean the device's resistance would vary according to the amount of charge that passed through it. And it would remember that resistance value even after the current was turned off. He proved that no combination of non-linear resistors, capacitors and inductors could duplicate the properties of a memristor ; therefore the memristor is truly fundamental [6].

\section{Modelling of Memristors}

The memristor is formally defined as a two-terminal element in which the magnetic flux $\Phi_{m}$ between the terminals is a function of the amount of electric charge $q$ that has passed through the device. Each memristor is characterized by its memristance function describing the charge-dependent rate of change of flux with charge [7].

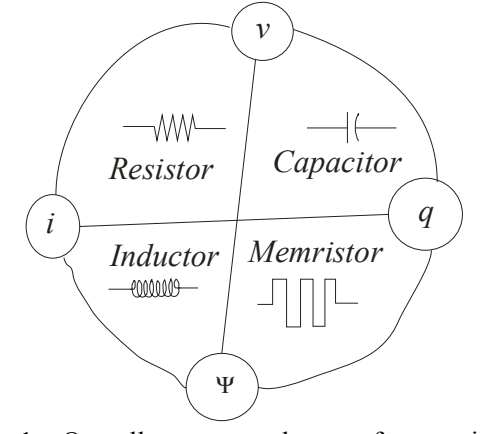

Fig. 1 : Overall component layout of a memristor

The $v-i$ relationships between the various elements is given by

Resistor : $d v=R d i$

Inductor : $d \varphi=L d i$

Capacitor : $d q=C d v$

But, in a memristor, it is the combination of all the three network elements, i.e., $R, L, C$. Thus, in a memristor, the modelling equation is given by $d \varphi=M d q$ [8].

Noting from Faraday's law of induction that magnetic flux is simply the time integral of voltage and charge is the time integral of current, we may write the more convenient form

$$
M(q(t))=\frac{d \phi_{m} / d t}{d q / d t}=\frac{v(t)}{i(t)}
$$

It can be inferred from this equation that memristance is simply charge-dependent resistance. If $M(q(t))$ is a constant, then we obtain Ohm's Law $R(t)=v(t) / i(t)$. If $M(q(t))$ is nontrivial, however, the equation is not equivalent because $q(t)$ and $M(q(t))$ will vary with time. Solving for voltage as a function of time we obtain [9]

$$
v(t)=i(t) M(q(t))
$$

This equation reveals that memristance defines a linear relationship between current and voltage, as long as charge does not vary. Of course, non-zero current implies time varying charge. Alternating current, however, may reveal the linear dependence in circuit operation by inducing a measurable voltage without net charge movement - as long as the maximum change in q does not cause much change in $M$. Furthermore, the memristor is static if no current is applied. If $i(t)=0$, we find $v(t)=0$ and $M(t)$ is constant. This is the essence of the memory effect. The power consumption characteristic recalls that of a resistor, $i^{2} R \&$ is given by [10]

$$
P(t)=i(t) v(t)=I^{2}(t) . M(q(t))
$$

As long as $M(q(t))$ varies little, such as under alternating currents, the memristor will appear as a resistor. If $M(q(t))$ increases rapidly, however, current and power consumption will quickly stop [11].

\section{Magnetic Flux in A Passive Device}

In circuit theory, magnetic flux $\Phi_{\mathrm{m}}$ typically relates to Faraday's law of induction, which states that the voltage in terms of electric field potential gained around a loop (electromotive force) equals the negative derivative of the flux through the loop [1] as

$$
\varepsilon=\frac{-d \Phi_{m}}{d t}
$$

This notion may be extended by analogy to a single passive device. If the circuit is composed of passive devices, then the total flux is equal to the sum of the flux components due to each device. For example, a simple wire loop with low resistance will have high flux linkage to an applied field as little flux is "induced" in the opposite direction. Voltage for passive devices is evaluated in terms of energy lost by a unit of charge as [2]

$$
v=\frac{d \Phi_{m}}{d t}
$$

Where $\Phi_{m}=\int \mathrm{V} d t$

Observing that $\Phi_{m}$ is simply equal to the integral of the potential drop between two points, we find that it may readily be calculated, for example by an operational amplifier configured as an integrator [3].

Two un-intuitive concepts are at play, viz., the magnetic flux $\&$ the response. The magnetic flux is generated by a 
resistance in opposition to an applied field or electromotive force. In the absence of resistance, flux due to constant EMF increases indefinitely. The opposing flux induced in a resistor must also increase indefinitely so their sum remains finite. Any appropriate response to applied voltage may be called "magnetic flux" [4].

The upshot is that a passive element may relate some variable to flux without storing a magnetic field. Indeed, a memristor always appears instantaneously as a resistor. As shown above, assuming non-negative resistance, at any instant it is dissipating power from an applied EMF and thus has no outlet to dissipate a stored field into the circuit. This contrasts with an inductor, for which a magnetic field stores all energy originating in the potential across its terminals, later releasing it as an electromotive force within the circuit [5].

\section{Operation Of Memristor As A Switch}

For some memristors, applied current or voltage will cause a great change in resistance. Such devices may be characterized as switches by investigating the time and energy that must be spent in order to achieve a desired change in resistance. Here we will assume that the applied voltage remains constant and solve for the energy dissipation during a single switching event. For a memristor to switch from $R_{o n}$ to $R_{\text {off }}$ in time $T_{\text {on }}$ to $T_{\text {off }}$, the charge must change by [6]

$$
E_{\text {switch }}=V^{2} \int_{T_{O f f}}^{T_{O_{n}}} \frac{d t}{M(q(t))}=V^{2} \int_{Q_{o f f}}^{Q_{O_{n}}} \frac{d q}{I(q) M(q)}=V^{2} \int_{Q_{\text {off }}}^{Q_{O_{n}}} \frac{d q}{V(q)}=V \Delta Q
$$

To arrive at the final expression, substitute $V=i(q) M(q)$, and then $\int d q / V=\Delta Q / V$ for constant $V$. This power characteristic differs fundamentally from that of a metal oxide semiconductor transistor, which is a capacitor-based device. Unlike the transistor, the final state of the memristor in terms of charge does not depend on bias voltage [7].

\section{IMPLEMENTATION OF MEMRISTORS}

Even before Professor Chua postulated the existence of memristor, many researchers were reporting what they called "anomalous" current-voltage behavior in the micrometer-scale devices they had built out of unconventional materials, like polymers and metal oxides. But the idiosyncrasies were usually ascribed to some mystery electrochemical reaction, electrical breakdown, or other spurious phenomenon attributed to the high voltages that researchers were applying to their devices. As it turns out, a great many of these reports were unrecognized examples of memristance. After Chua theorized the memristor out of the mathematical ether, no one knew how to build one [8].

Now, 37 years later, electronics have finally gotten small enough to reveal the secrets of that fourth element. On April 30, 2008 a team at HP Labs announced the development of a switching memristor. Based on a thin film of titanium dioxide, it has a regime of operation with an approximately linear charge-resistance [9].
And on May $1^{\text {st }}$ Hewlett-Packard researcher, R. Stanley William and his group of nearly hundred scientists and researchers published the details of the memristor in the journal Nature.

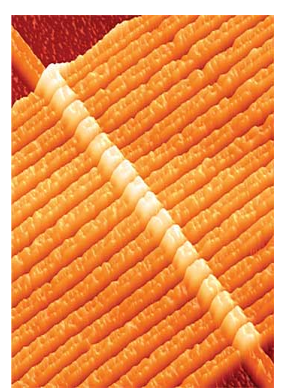

Fig. 2 : Cross-bar architecture of a memristor's structure, shown in a scanning tunneling process

\section{Why The Process of FABricAting the MeMristor TOOK SO LONG PROCESS}

It was all about scale. We now know that memristance is an intrinsic property of any electronic circuit. Its existence could have been deduced by Gustav Kirchhoff or by James Clerk Maxwell, if either had considered non-linear circuits in the 1800 's. But the scales at which electronic devices have been built for most of the past two centuries have prevented experimental observation of the effect. It turns out that the influence of memristance obeys an inverse square law : memristance is a million times as important at the nanometer scale as it is at the micrometer scale, and it's essentially unobservable at the millimeter scale and larger. As smaller and smaller devices were being built, memristance became more noticeable and in some cases dominant. That's what accounts for all those strange results researchers have described. Memristance has been hidden in plain sight all along [10].

\section{Why Titanium Dioxide \& Not Any Other MATERial FOR MEMRISTOR}

HP labs were the first to understand that the hysteresis that was being observed in the I - V curves of a wide variety of materials and structures was actually the result of memristance and something more general that can be called 'memristive behavior'. They then went on to create an elementary circuit model that was defined by exactly the same mathematical equations as those predicted by Chua for the memristor, with the exception that this model had an upper bound to the resistance (which means that at large bias or long times, it is a memristive device). It was then showed that this simple model could reproduce a wide variety of eccentric and complex I - V curves that have been observed and reported over the years by many researchers, including ourselves. Most of these did not look much like the 'figure 8 ' curves of Chua in his paper, but rather ' $S$ ' and ' $N$ ' curves that have erroneously been attributed to negative differential resistance, which is one reason why the connection to memristive behavior had not been made earlier. A highly simplified form appropriate for a general audience journal like Nature or for a basic undergraduate course, the equations for the drift of oxygen vacancies in $\mathrm{TiO}_{2}$ and their influence 
on the electronic conduction in the material were also identical with our equivalent circuit model, and thus Chua's memristor equations was also shown [11].

From this, for the first time a formula was written down for the memristance of a device in terms of material and geometrical properties of the device (just as the resistance is the resistivity of the material times the length divided by the cross sectional area of the resistor). This memristance formula immediately showed that the size of the most important term in the memristance gets larger the smaller the device - thus showing that it was not very important for micron-scale electronics but is becoming very important for nanoscale device. In the Fig. 3 shown, researchers removed transistors from the bottom layer of this silicon-based chip (shown in yellow and blue) and replaced them with fewer memristors in the top layer (shown in red). Memristors can do the work of transistors but require less power and space. According to HP, they could revolutionize integrated circuits, an memory technology [1].

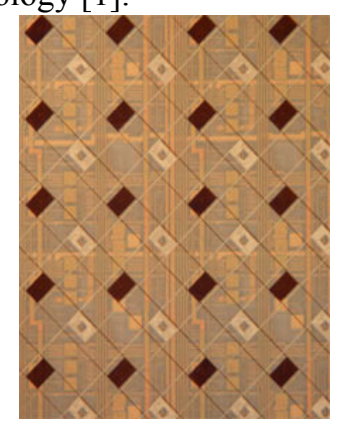

Fig. 3 : Researchers removed transistors from the bottom layer of this silicon-based chip

\section{The Titanium Dioxide Based Memristor : How The EXISTANCE OF MEMRISTIVE BEHAVIOUR IN TITANIUM DIOXIDE WAS DISCOVERED}

In 1995, Wi was recruited to HP Labs to start up a fundamental research group that had been proposed by David Packard. The research was to be in the field of molecular-scale electronics and something useful was expected to come out in about 10 years [2].

They had decided to take on Moore's Law. The existing semiconductor road map at that time didn't extend past 2010. The critical feature size for the transistors on an integrated circuit was 350 nanometers; they had a long way to go before atomic sizes would become a limitation. And yet, the eventual end of Moore's Law was obvious. Someday semiconductor researchers would have to confront physics-based limits to their relentless descent into the infinitesimal, if for no other reason than that a transistor cannot be smaller than an atom. Today, the smallest components of transistors on integrated circuits are roughly $45 \mathrm{~nm}$ wide, or about $220 \mathrm{si}$ atoms [3].

They started their work with Phil Kuekes, the creative force behind the Teramac (tera - operation - per - second with a multi - architecture computer) - an experimental supercomputer built at HP Labs primarily from defective parts, just to show it could be done. He gave them the idea to build an architecture that would work even if a substantial number of the individual devices in the circuit were dead on arrival. They didn't know what those devices would be, but their goal was electronics that would keep improving even after the devices got so small that defective ones would become common. They then started to speculate about what this mystery nano-device would be [4].

They were designing something that wouldn't even be relevant for another 10 to 15 years. It was possible that by then devices would have shrunk down to the molecular scale envisioned by David Packard or perhaps even be molecules. They could think of no better way to anticipate this than by mimicking the Teramac at the nanoscale. The simplest abstraction of the Teramac architecture was the crossbar, which has since become the de-facto standard for nanoscale circuits because of its simplicity, adaptability, and redundancy [5].

The crossbar is an array of perpendicular wires. Anywhere two wires cross, they are connected by a switch. To connect a horizontal wire to a vertical wire at any point on the grid, you must close the switch between them. The idea was to open and close these switches by applying voltages to the ends of the wires. Crossbar switches are most famously used in information processing applications such as telephony and packet switching, but they are also used in applications such as mechanical sorting machines with inputs [6].

One of the major impediments for prior crossbar memory research was the small off-to-on resistance ratio of the switches (40 years of research had never produced anything surpassing a factor of 2 or 3). By comparison, modern transistors have an off-to-on resistance ratio of 10000 to 1 . It was calculated that to get a high-performance memory, the switches were to be made with a resistance ratio of at least 1000 to 1 . In other words, in its off state, a switch had to be 1000 times as resistive to the flow of current as it was in its on state [7].

What mechanism could possibly give a nanometer-scale device a three-orders-of-magnitude resistance ratio? The answer was found in scanning tunneling microscopy (STM). A tunneling microscope generates atomic-resolution images by scanning a very sharp needle across a surface and measuring the electric current that flows between the atoms at the tip of the needle and the surface the needle is probing. The general rule of thumb in STM is that moving that tip $0.1 \mathrm{~nm}$ closer to a surface increases the tunneling current by one order of magnitude. Some similar mechanism was needed by which the effective spacing between two wires in the crossbar could be changed by $0.3 \mathrm{~nm}$. This would then yield a ratio of 1000:1 electrical switching ratio that was needed [8].

To adhere to the constraints of a material that could change its physical dimensions in the above manner, it was decided to build a device in the realm of molecular devices. The device was like a tiny sandwich. Two platinum electrodes (the intersecting wires of the crossbar junction) functioned as the "bread" on either end of the device. The surface of the bottom platinum wire was oxidized to make an extremely thin layer of platinum dioxide, which is highly conducting. Next, a dense film was assembled, only one molecule thick, of specially designed switching molecules. Over this "monolayer" we deposited a 2 - to 3 - nm layer of titanium metal, which bonds strongly to the molecules and were 
intended to glue them together. The final layer was the top platinum electrode [9].

The molecules were supposed to be the actual switches. An enormous number of these devices were built experimenting with a wide variety of exotic molecules and configurations, including, Heath and Stoddart's devices using silicon electrodes. They had worked, but not well enough for technological applications: the off-to-on resistance ratio was only a factor of 10 , the switching was slow, and the devices tended to switch themselves off after 15 minutes. Platinum devices used yielded results that were frustrating. When a switch worked, it was spectacular: our off-to-on resistance ratios shot past the 1000 mark, the devices switched too fast for it to be even measured, and having switched, the device's resistance state remained stable for years. But, the results were inconsistent. Worse yet, the success or failure of a device never seemed to depend on the same thing. There was no physical model for how these devices worked. It was late into 2002, six years into the research, there was something that worked, but it couldn't be figured out why, it couldn't be modeled or engineered [10].

That's when Greg Snider, who had worked with Kuekes on the Teramac, brought the Chua memristor paper from the September 1971 IEEE Transactions on Circuits Theory. Chua's paper had included a graph that looked suspiciously similar to the experimental data that were being collected. The graph described the current-voltage (I - V) characteristics that Chua had plotted for his memristor. Chua had called them "pinched-hysteresis loops", I - V characteristics for the platinum device were called "bow ties." A pinched hysteresis loop looks like a diagonal infinity symbol with the center at the zero axis, when plotted on a graph of current against voltage. The voltage is first increased from zero to a positive maximum value, then decreased to a minimum negative value and finally returned to zero. The bow ties were nearly identical. That's not all [11].

The total change in the resistance measured in the devices also depended on how long the voltage was applied : the longer applied a positive voltage, the lower the resistance until it reached a minimum value. And the longer applied negative voltage is, the higher the resistance became until it reached a maximum limiting value. When the application of voltage was stopped, whatever resistance characterized the device was frozen in place, until it was reset once again by applying a voltage. The loop in the I - V curve is called hysteresis, and this behavior is startlingly similar to how synapses operate: synaptic connections between neurons can be made stronger or weaker depending on the polarity, strength, and length of a chemical or electrical signal. That's not the kind of behavior you find in today's circuits [1].

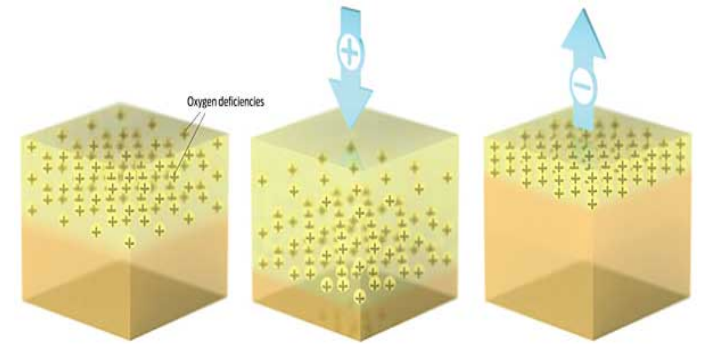

Fig. 4 : Memristor structures
Looking at Chua's graphs gave a big clue that memristance had something to do with the switches. But how ? Why should our molecular junctions have anything to do with the relationship between charge and magnetic flux? By 2004, a little surgery on the little sandwiches was carried out. A gadget was built that ripped the tiny devices open so that one could peer inside them and do some forensics. When prided apart, the little sandwiches separated at their weakest point : the molecule layer. For the first time, a good look at what was going on inside was visible. What was seen was not what was had built. Recall that it was a sandwich with two platinum electrodes as the bread and filled with three layers : the platinum dioxide, the monolayer film of switching molecules, and the film of titanium [2].

But that's not what was found. Under the molecular layer, instead of platinum dioxide, there was only pure platinum. Above the molecular layer, instead of titanium, we found an unexpected and unusual layer of titanium dioxide. The titanium had sucked the oxygen right out of the platinum dioxide! The oxygen atoms had somehow migrated through the molecules and been consumed by the titanium. This was especially surprising because the switching molecules had not been significantly perturbed by this event-they were intact and well ordered, which convinced us that they must be doing something important in the device. The chemical structure of our devices was not at all what we had thought it was [3].

The titanium dioxide - a stable compound found in sunscreen and white paint-was not just regular titanium dioxide. It had split itself up into two chemically different layers. Adjacent to the molecules, the oxide was stoichiometric $\mathrm{TiO}_{2}$, meaning the ratio of oxygen to titanium was perfect, exactly 2 to 1 . But closer to the top platinum electrode, the titanium dioxide was missing a tiny amount of its oxygen, between 2 and 3 percent. We called this oxygen-deficient titanium dioxide $\mathrm{TiO}_{2-x}$, where $x$ is about 0.05 . Because of this misunderstanding, the experiment was conducted backward. Every time a switching model was created, the polarity was reversed. In other words, it was predicted that a positive voltage would switch the device off and a negative voltage would switch it on. In fact, exactly the opposite was true. It was time to get to know titanium dioxide a lot better. By the end of the month, the pieces had fallen into place. It was finally clear how the device worked and how they had a memristor [4].

\section{HOW THE MEMRISTORS WORK ?}

The exotic molecule monolayer in the middle of the sandwich had nothing to do with the actual switching. Instead, what it did was control the flow of oxygen from the platinum dioxide into the titanium to produce the fairly uniform layers of $\mathrm{TiO}_{2}$ and $\mathrm{TiO}_{2-\mathrm{x}}$. The key to the switching was this bilayer of the two different titanium dioxides. The $\mathrm{TiO}_{2}$ is electrically insulating (actually a semiconductor), but the $\mathrm{TiO}_{2-\mathrm{x}}$ is conductive, because its oxygen vacancies are donors of electrons, which makes the vacancies themselves positively charged, they can be pushed up and down at will in the titanium dioxide material because they are electrically 
charged [5].

If a positive voltage is applied to the top electrode of the device, it will repel the (also positive) oxygen vacancies in the $\mathrm{TiO}_{2-x}$ layer down into the pure $\mathrm{TiO}_{2}$ layer. That turns the $\mathrm{TiO}_{2}$ layer into $\mathrm{TiO}_{2-x}$ and makes it conductive, thus turning the device on. A negative voltage has the opposite effect: the vacancies are attracted upward and back out of the $\mathrm{TiO}_{2}$ and thus the thickness of the $\mathrm{TiO}_{2}$ layer increases and the device turns off. This switching polarity is what we had been seeing for years but had been unable to explain [6].

Two important equations were then written - one equation detailing the relationship between current and voltage for this equivalent circuit, and another equation describing how the application of the voltage causes the vacancies to move - thereby writing down, for the first time, an equation for memristance in terms of the physical properties of a material. This provided a unique insight. Memristance arises in a semiconductor when both electrons and charged dopants are forced to move simultaneously by applying a voltage to the system. The memristance did not actually involve magnetism in this case; the integral over the voltage reflected how far the dopants had moved and thus how much the resistance of the device had changed [7].

Finally a model was made to engineer the switches, which were now positively identified as memristors. It was then declared that we had to take the molecule monolayers out of our devices. It was important to find the exact amounts of titanium and oxygen to get the two layers to do their respective jobs. A month later, it worked. Not only did they have working devices, but were also able to improve and change their characteristics at will [8].

But here is the real triumph. The resistance of these devices stayed constant whether we turned off the voltage or just read their states (interrogating them with a voltage so small it left the resistance unchanged). The oxygen vacancies didn't roam around; they remained absolutely immobile until we again applied a positive or negative voltage. That's memristance: the devices remembered their current history. Chua's mythical memristor was coaxed out off the page [9].

\section{Resistance to MemRISTANCE}

Introducing a new fundamental circuit element earned $\mathrm{R}$. Stanley Williams some grief along with his newfound fame. After the NATURE article appeared in May, online comments pages boiled over with skepticism. "Is this a hoax?" someone asked on the Wikipedia memristor page on 30 April 2008, the day the news broke, in one of the milder statements of disbelief. Seven months later, the debate continues [4].

Skeptics argue that the memristor is not a fourth fundamental circuit element but an example of bad science. The crux of their argument rests on two fundamental misunderstandings: first, skeptics overlook the expanded design space that arises from working with nonlinear circuit elements. The second and more profound misunderstanding concerns Leon Chua's mathematical definition of a memristor [10].

At first, most people_-including Williams — assumed that
Chua defined memristance strictly as the relationship between electric charge and magnetic flux. However, the actual definition of memristance is more general. Linking electric charge and magnetic flux is one way to satisfy the definition, but it's not the only one. In fact, it turns out you can bypass magnetic interaction altogether.

Chua's general memristance definition has two parts: The first equation defines how the memristor's voltage depends on current and a "state variable" - that is, a quantity that measures some physical property of a device, like the length of a column of mercury in a thermometer [11].

The column's length correlates to the thermometer's temperature, and adding or removing heat makes the column longer or shorter. In Williams's memristor, the state variable is the thickness of the stoichiometric titanium dioxide in the switch; increasing or decreasing that thickness causes the device's resistance to increase or decrease.

The second equation expresses how the changing state variable (the $\mathrm{TiO}_{2}$ 's thickness) depends on the amount of charge flowing through the device. In Williams's memristor, the $\mathrm{TiO}_{2}$ 's thickness depends on the distribution of the oxygen vacancies throughout the material. Here is what you need to remember [1] :

- A magnetic interaction is not necessary for memristance.

- In nonlinear circuit elements, memristance is not the same thing as nonlinear resistance.

- Because no combination of passive devices can reproduce the properties of a memristor, memristance is a fundamental circuit quantity.

Williams himself is sanguine about the memristor's reputation. "A hundred years after Einstein proposed his theory of relativity," he says, shrugging, "you still have some people arguing against it." [2]

\section{Timeline of Memristor And Memristive Systems DEVELOPMENTS}

Since the beginning of the invention of the transistor by William Shockley \& his associates, a number of changes have taken place in the transistor miniaturization [1]-[11].

To start with, we give a brief review starting from the $70 \mathrm{~s}$. 1971

Leon Chua, a professor at UC Berkeley, postulates a new two-terminal circuit element characterized by a relationship between charge and flux linkage as a fourth fundamental circuit element in the article "Memristor-the Missing Circuit Element" published in IEEE Transactions on Circuit Theory. 1976

Leon Chua and his student Sung Mo Kang publish a paper entitled "Memristive Devices and Systems" in the Proceedings of the IEEE generalizing the theory of memristors and memristive systems including a property of zero crossing in the Lissajous curve characterizing current vs. voltage behavior.

1986

Robert Johnson and Stanford Ovshinsky receive U.S. Patent 4,597,162 describing manufacturing of a 2-terminal reconfigurable resistance switching array based on phase changing materials. While distinct from memristor behavior 
some of the basic elements later used by Stan Williams's group such as the use of crossbar architecture and the basic use of a 2-terminal resistance switch are found in this patent.

1998

Bhagwat Swaroop, William West, Gregory Martinez, Michael Kozicki, and Lex Akers publish a paper entitled "Programmable Current Mode Hebbian Learning Neural Network Using Programmable Metallization Cell" in the Proceedings of the IEEE International Symposium on Circuits and Systems, (vol. 3, pp. 33-36, 1998), demonstrating that the complexity of an artificial synapse can be minimized by using an ionic programmable resistance device. (Jun. 3)

2005

Darrell Rinerson, Christophe Chevallier, Steven Longcor, Wayne Kinney, Edmond Ward, and Steve Kuo-Ren Hsia receive U.S. Patent 6,870,755 (assigned to Unity Semiconductor) including basic patent claims to reversible 2-terminal resistance switching materials based on metal oxides (Mar. 22).

\section{8}

Dmitri Strukov, Gregory Snider, Duncan Stewart, and Stan Williams, of HP Labs, publish an article in Nature "The missing memristor found" identifying a link between the 2-terminal resistance switching behavior found in nanoscale systems and Leon Chua's memristor. (May 1) Leon Chua, Stan Williams, Greg Snider, Rainer Waser, Wolfgang Porod, Massimiliano Di Ventra, and Blaise Mouttet speak at a Symposium on Memristors and Memristive Systems held at UC Berkeley. Discussion includes the theoretical foundations of memristors and memristive systems of Leon Chua and Sung Mo Kang and the prospects of memristors for RRAM and neuromorphic electronic architectures (Nov. 21). 2009

Massimiliano Di Ventra, Yuriy V. Pershin, Leon O. Chua submit an article in arxiv.org entitled "Circuit elements with memory: memristors, memcapacitors and meminductors" which extends the notion of memristive systems to capacitive and inductive elements, namely capacitors and inductors whose properties depend on the state and history of the system. (January 23, 2009) Blaise Mouttet published a Google knol article entitled: "An Introduction to Memimpedance and Memadmittance Systems Analysis" which is an explanation on "Circuit elements with memory: memristors, memcapacitors and meminductors" and Chua's memristor paper. (Jan. 30, 2009)

\section{ApPliCATIONS}

Emulating the behavior of a single memristor, Chua showed, requires a circuit with at least 15 transistors and other passive elements. The implications are extraordinary: just imagine how many kinds of circuits could be supercharged by replacing a handful of transistors with one single memristor. A few applications of memristors are [1]-[4]:

NVRAM : It stands for non-volatile random access memory. In its initial state, a crossbar memory has only open switches, and no information is stored. But once you start closing switches, you can store vast amounts of information compactly and efficiently. Because memristors remember their state, they can store data indefinitely, using energy only when you toggle or read the state of a switch, unlike the capacitors in conventional DRAM, which will lose their stored charge if the power to the chip is turned off. The NVRAM made with the types of memristor materials that are currently being studied by many groups around the world could be a strong competitor to the flash memory market in about five years. The great thing is that the various metal oxides that have been identified as having a memory function are highly compatible with present chip fabrication facilities, so they can be made in existing foundries without a lot of changes being required [5]-[8].

FPGA'S: A paper was published last year by Greg Snider and William Stanley showing that memristors could vastly improve one type of processing circuit, called a field-programmable gate array, or FPGA. By replacing several specific transistors with a crossbar of memristors, we showed that the circuit could be shrunk by nearly a factor of 10 in area and improved in terms of its speed relative to power-consumption performance. Right now, a prototype of this circuit is being tested in the HP labs. And memristors are by no means hard to fabricate. The titanium dioxide structure can be made in any semiconductor FAB currently in existence. The primary limitation to manufacturing hybrid chips with memristors is that today only a small number of people on Earth have any idea of how to design circuits containing memristors. It must be emphasize here that memristors will never eliminate the need for transistors : passive devices and circuits require active devices like transistors to supply energy [9]-[11].

MIMICING THE BRAIN (neural network) : There is a similarity of memristor behavior to that of synapses. Right now new circuits are being designed that mimic aspects of the brain. The neurons are implemented with transistors, the axons are the nano-wires in the crossbar, and the synapses are the memristors at the cross points. A circuit like this could perform real-time data analysis for multiple sensors. Think about it: an intelligent physical infrastructure that could provide structural assessment monitoring for bridges. How much money - and how many lives — could be saved ? [1]

ANALOG PARALLEL COMPS : Low-cost, low - energy consuming computers that are much better suited to parallel processing the massive streams of real-time data feeds that are required to analyze, model, and diagnose many of our planet's toughest problems - like global warming. Instead of (or in addition to) supercomputers in the cloud, memristive technologies could be deployed in distributed and embedded systems. These memristors can be turned into "integrated circuits that remember information, consume far less power than existing devices, and may someday learn from past behavior. An analog computer is a computational device in which the problem variables are represented as continuous, varying physical quantities. An analog computer implements a model of the system being studied. The physical form of the analog may be functionally similar to that of the system, but more often the analogy is based solely upon the mathematical equivalence of the interdependence of the computer variables 
and the variables in the physical system [10].

\section{CONCLUSIONS}

This paper presented a brief review of the recent advanced technologies that lead to the development of the element, viz., the memristor \& its wide-spread applications.

\section{REFERENCES}

[1] Leon Chua, "Memristor-the Missing Circuit Element", Proc. IEEE Transactions on Circuit Theory, 1971.

[2] Leon Chua and his student Sung Mo Kang, "Memristive Devices and Systems : Generalizing the theory of memristors and memristive systems including a property of zero crossing in the Lissajous curve characterizing current vs. voltage behavior, Proceedings of the IEEE, 1976.

[3] Robert Johnson and Stanford Ovshinsky, U.S. Patent 4,597,162 "Manufacturing of a 2-terminal reconfigurable resistance switching array based on phase changing materials" patent, 1986.

[4] Bhagwat Swaroop, William West, Gregory Martinez, Michael Kozicki, and Lex Akers, "Programmable Current Mode Hebbian Learning Neural Network Using Programmable Metallization Cell", Proceedings of the IEEE International Symposium on Circuits and Systems, Vol. 3 , pp 33-36, 1998, Jun. 3.

[5] Darrell Rinerson, Christophe Chevallier, Steven Longcor, Wayne Kinney, Edmond Ward, and Steve Kuo-Ren Hsia, U.S. Patent $6,870,755$ (assigned to Unity Semiconductor) including basic patent claims to reversible 2-terminal resistance switching materials based on metal oxides, Mar. 22, 2005.

[6] Dmitri Strukov, Gregory Snider, Duncan Stewart, and Stan Williams, of HP Labs, Proc. of the Nature, "The missing memristor found" identifying a link between the 2-terminal resistance switching behavior found in nanoscale systems and Leon Chua's memristor, May 1, 2008.

[7] Leon Chua, Stan Williams, Greg Snider, Rainer Waser, Wolfgang Porod, Massimiliano Di Ventra, and Blaise Mouttet, Symposium on Memristors and Memristive Systems held at UC Berkeley. Discussion includes the theoretical foundations of memristors and memristive systems of Leon Chua and Sung Mo Kang and the prospects of memristors for RRAM and neuromorphic electronic architectures, Nov. 21.

[8] http://www.howstuffworks.com

[9] Massimiliano Di Ventra, Yuriy V. Pershin, Leon O. Chua submit an article in arXiv.org entitled, "Circuit elements with memory: memristors, memcapacitors and meminductors" which extends the notion of memristive systems to capacitive and inductive elements, namely capacitors and inductors whose properties depend on the state and history of the system, Jan. 23, 2009.

[10] Blaise Mouttet published a Google knol article entitled: "An Introduction to Memimpedance and Memadmittance Systems Analysis" which is an explanation on "Circuit elements with memory: memristors, memcapacitors and meminductors" and Chua's memristor paper, Jan. 30, 2009.

[11] http://www.en.wikipedia.org

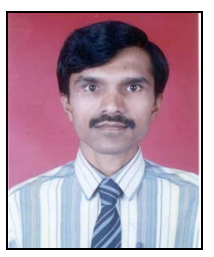

Dr. T.C. Manjunath born in Bangalore, Karnataka, India on Feb. 6, 1967 received the B.E. Degree (Bachelor of Engg.) from R.V. College of Engg. (Bangalore University) in 1989 with First Class and M.E. degree (Master of Engg.) from L.D. College of Engg. (Gujarat University) in 1995 in First Class with Distinction and Ph.D. from the prestigious \& renowned Indian Institute of Technology Bombay (IIT Bombay) in the year 2007, respectively. He have got a teaching experience of nearly 20 long years in various engineering colleges all over the country (Karnataka, Tamilnadu, Gujarat, Maharashtra) and is currently working as Professor and Head of the Department of Electronics and Communication Engineering in Rajarajeshwari College of Engg. in Bangalore, Karnataka, India. He also worked as a Project Assistant and as a Research Engineer in the Systems and Control Engineering (IIT Bombay, India) and worked on control of space launch vehicles using FOS feedback technique. He has published a large number of papers in the various National, International journals and
Conferences and published three textbooks on Robotics, one of which has gone upto the fourth edition, titled, 'Fast Track to Robotics' and the other, which has gone upto the fifth edition, titled, 'Fundamentals of Robotics' in 2 volumes, Vol.-1 and Vol.-2 along with a CD which contains about $150 \mathrm{C} /$ $\mathrm{C}++$ programs for performing various simulations on robotics. He has also published a research monograph along with his faculty advisors in the International level from the Springer-Verlag publishers based on his Ph.D. thesis topic titled, "Modeling, Control and Implementation of Smart Structures", Vol. 350, LNCIS, costing 79.95 Euros. He was a member of IEEE since 7 years, was a SPIE student member and IOP student member for 4 years, life member of ISSS (India), life member of the ISTE (India), life member of ISOI (India), life member of SSI (India) and life member of the CSI (India) and life member cum fellow of the IETE (India). He has also presented a number of guest lectures, various seminars and participated in more than a dozen CEP / DEP courses, seminars, workshops, symposiums in the various parts of the country in different institutions and also conducted a few of them. He has visited Singapore, Russia, United States of America and Australia for the presentation of his research papers in various international conferences. His biography was published in $23^{\text {rd }}$ edition of Marquis's Who's Who in the World in the 2006 issue. He has also guided more than 2 dozen projects (B.Tech. / M.Tech.) in various engineering colleges where he has worked so far. Many of his guided projects, interviews have appeared in various national newspapers and magazines. He have also reviewed many research papers for the various international conferences such as IEEE IECON-06, IEEE-ISIE-2007, NSC-07, IEEE-WCSN-07, IEEE-PSACO2008, etc., and has also reviewed many journal papers for journals such as IJAMT, AJC, etc... He has also given many invited talks, key note speeches / plenary lecturers in various national \& international conferences and chaired many sessions. He has also conducted / organized / convened / coordinated more than one dozen courses / workshops / technical paper fests, students symposiums, etc., in various engineering colleges where he has worked. His Ph.D. research work was based on the mathematical modeling, control and implementation of smart structures and its applications to Robotics, Aerospace \& Civil Engg. His current research interests are in the area of Robotics \& AI, Smart Structures, Control systems. 